\title{
Advanced Test of Attention in Children with Attention-Deficit/Hyperactivity Disorder in Japan for Evaluation of Methylphenidate and Atomoxetine Effects
}

\author{
Toru Fujioka ${ }^{1,2}$, Shinichiro Takiguchi ${ }^{3}$, Chiho Yatsuga ${ }^{4}$, Michio Hiratani $^{5}$, Kang-E M Hong ${ }^{6}$, Min-Sup Shin ${ }^{7}$, \\ Sungzoon $\mathrm{Cho}^{8}$, Hirotaka Kosaka, ${ }^{1,2}$, Akemi Tomoda ${ }^{1}$ \\ 'Research Center for Child Mental Development, University of Fukui, Fukui, ${ }^{2}$ Department of Neuropsychiatry, University of Fukui, Fukui, \\ ${ }^{3}$ Advanced Biomedical Sciences Course, Graduate School of Medical Sciences, University of Fukui, Fukui, ${ }^{4}$ National Hospital Organization \\ Hizen Psychiatric Center, Saga, ${ }^{5}$ Hiratani Clinic for Developmental Disorders of Children, Fukui, Japan, ${ }^{6}$ Department of Psychiatry, \\ ${ }^{7}$ Department of Child and Adolescent Psychiatry, Seoul National University College of Medicine, ${ }^{8}$ Department of Industrial Engineering, \\ Seoul National University College of Engineering, Seoul, Korea
}

\begin{abstract}
Objective: This study was conducted to validate the Advanced Test of Attention (ATA) of the visual attention version of Japanese children with attention deficit/hyperactivity disorder (ADHD) and to evaluate the efficacy of methylphenidate (OROS-MPH) and atomoxetine medications.

Methods: To assess pharmacotherapy efficacy, the visual version of ATA was administered to 42 children with ADHD. Results were assessed using discriminant analysis, ANOVA for indices of ATA before and after medication treatment, and correlation analysis between the improvement of indices of ATA and clinical symptoms during medication treatment.

Results: Discriminant analysis showed that $69.0 \%$ of ADHD children were assigned correctly. The T score of commission errors increased as the trial progressed on the medication-off condition. T scores of commission errors and standard deviation of response times on medication-on condition were low compared to the medication-off condition. A few significant correlations were found between the improvements of indices of ATA and ADHD-Rating Scale (RS) during treatment.

Conclusion: The performance of the visual version of ATA on medication-off condition reflected the features of ADHD. Furthermore, the medication treatment effects were confirmed sufficiently. In addition, results suggest that indices of ATA reflected aspects of ADHD symptoms that are difficult to elucidate for ADHD-RS. For assessing symptoms and effects of medical treatment in children with ADHD, ATA might be a useful assessment tool.
\end{abstract}

KEY WORDS: Attention deficit hyperactivity disorder; Advanced Test of Attention; Commission error; Standard deviation of response times; Methylphenidate; Atomoxetine.

\section{INTRODUCTION}

Attention-deficit/hyperactivity disorder (ADHD) is characterized by a persistent pattern of inattention and/or hyperactivity-impulsivity that interferes with functioning or development in more than one setting, according to the Diagnostic and Statistical Manual of Mental Disorders fifth edition (DSM-5). ${ }^{1)}$ If left untreated, ADHD, which has been estimated to affect about $5 \%$ of school-aged children, ${ }^{1)}$ can influence children's studies, family settings,

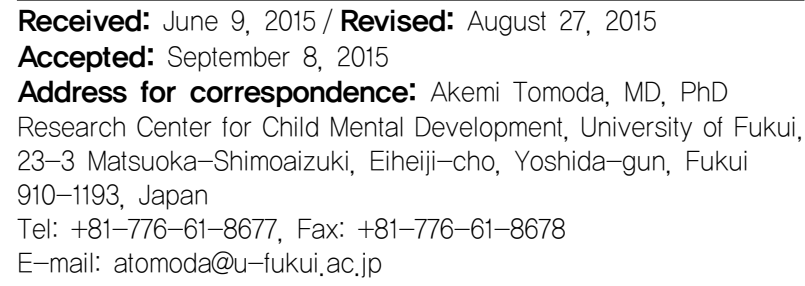

and social life.

Recent neuropsychological reports have described that ADHD is likely to be attributable to impairment of executive functions in the frontal lobe, and especially in the prefrontal cortex. ${ }^{2,3)}$ Executive function is the cognitive management of the human brain, responsible for planning, working memory, impulse control, inhibition, and shifting set as well as the initiation and monitoring of action. ${ }^{2,4)}$ Children and adolescents with ADHD experience much more difficulty related to these functions in their daily life than people without symptoms of ADHD.

To treat these difficulties, pharmacological therapy is widely used. Only osmotic release oral system methylphenidate (OROS-MPH) and atomoxetine (ATX) are approved for the treatment of ADHD in Japan. MPH, which is known to block the dopamine transporter and enhance dopamine release, ${ }^{5,6)}$ has been shown to affect brain func-

(c) This is an Open-Access article distributed under the terms of the Creative Commons Attribution Non-Commercial License (http://creativecommons.org/licenses/by-nc/4.0) which permits unrestricted non-commercial use, distribution, and reproduction in any medium, provided the original work is properly cited. 
Table 1. Mean chronological age (CA) and FIQ/FSIQ of each group

\begin{tabular}{|c|c|c|c|}
\hline & All ADHD $(n=42)$ & Inattentive group $(n=17)$ & Combined group $(n=25)$ \\
\hline CA & $9.9 \pm 2.3(6.0-14.8)$ & $10.2 \pm 2.1(6.0-14.8)$ & $9.6 \pm 2.4(6.1-14.3)$ \\
\hline $\mathrm{FIQ/FSIQ}$ & $95.3 \pm 13.5(60.0-122.0)$ & $96.7 \pm 13.3(60.0-122.0)$ & $94.4 \pm 13.8(76.0-115.0)$ \\
\hline
\end{tabular}

Values are presented as mean \pm standard deviation (range).

IQ, intelligence quotient; FIQ/FSIQ, full or full-scale IQ; $A D H D$, attention deficit/hyperactivity disorder.

tions, ${ }^{7-9)}$ and to improve performance on executive function such as spatial working memory, response inhibition, and set-shift. ${ }^{5)}$ Actually, ATX, which functions as an inhibitor of norepinephrine uptake, is a long-acting medication. ${ }^{6)}$ Its effectiveness is shown to persist into the evening hours and until the next morning. ${ }^{6,10)}$ ATX also is reported to improve cognitive abilities that show a deficit attributable to ADHD. ${ }^{6}$

For assessing neurocognitive deficits of children and adolescents with ADHD, Continuous Performance Tests (CPTs) are often used. CPTs have demonstrated sensitivity to executive dysfunction, especially the attention system. ${ }^{11,12)}$ As a standardized CPT, the Advanced Test of Attention (ATA) was developed and sold in Korea. ${ }^{13)}$ ATA was standardized with Korean children without ADHD and the standard scores of indices are calculated after completion. For assessment of ADHD or evaluation of medications, although it is important to compare standard scores of the same age group, no standardized CPT exists in Japan.

The study aims were to validate ATA of the visual attention version in Japan, and to evaluate the efficacy of medication treatment (OROS-MPH and ATX) using ATA. Although the ATA consists of a visual test and an auditory test, auditory test is more difficult than visual test and less sensitivity in differentiating subject with ADHD and control. ${ }^{14)}$ Therefore, only a visual test was performed.

\section{METHODS}

\section{Participants}

The Ethics Committee of the University of Fukui approved the study protocol (25-109). The authors have no financial conflict of interest to declare in relation to this study. Informed consent was obtained from all children and their caregivers. Participants in this study were 42 (36 boys, 6 girls) Japanese children with ADHD (mean age \pm stadard deviation [SD], 9.9 \pm 2.3 years; range, 6-14 years), 17 were inattentive, and 25 were combined type ( 5 participants and 1 participant were female, respectively). The diagnosis was made by four child psychiatric specialists on DSM-5 according to information related to their
Table 2. Number of comorbidities of each group

\begin{tabular}{lcc|c}
\hline & $\begin{array}{c}\text { Whole } \\
(\mathrm{n}=42)\end{array}$ & $\begin{array}{c}\text { Inattentive } \\
\text { subtype } \\
(\mathrm{n}=17)\end{array}$ & $\begin{array}{c}\text { Combined } \\
\text { subtype } \\
(\mathrm{n}=25)\end{array}$ \\
\hline Comorbidity & & & \\
ASD & 23 & 7 & 16 \\
LD & 7 & 6 & 1 \\
ODD & 3 & 2 & 1 \\
CD & 1 & 0 & 1 \\
RAD & 1 & 0 & 1 \\
Medication treatment & & & 18 \\
OROS-MPH & 33 & 15 & 7 \\
ATX & 9 & 2 & 18 \\
\hline
\end{tabular}

ADHD, attention deficit/hyperactivity disorder; ASD, autism spectrum disorders; LD, learning disorders; ODD, oppositional defiant disorder; CD, conduct disorder; RAD, reactive attachment disorder; OROS-MPH, osmotic release oral system methylphenidate; ATX, atomoxetine.

developmental history and current clinical pictures from semi-structured interview to parents and behavioral observation. For children who were diagnosed previously, the above-mentioned specialists reassessed their symptoms on DSM-5. Thirteen children were examined using the Japanese version of the Wechsler Intelligence Scale for Children third edition (WISC-III; mean full intelligence quotient $[\mathrm{IQ}] \pm \mathrm{SD}, 98.2 \pm 11.4$; range, 75-115). Others were examined using the fourth edition (WISC-IV; mean full-scale $\mathrm{IQ} \pm \mathrm{SD}, 94.1 \pm 14.2$; range, 60-122). More detailed information is presented in Table 1.

Twenty-three children were also diagnosed as having autism spectrum disorders (ASDs), seven children as having learning disorders, three children as having oppositional defiant disorder, and one child as having conduct disorder and reactive attachment disorder.

Thirty-three children were treated with OROS-MPH; nine were treated with ATX. More detailed information for each subgroup is presented in Table 2.

\section{Measures}

\section{Advanced Test of Attention}

The ATA, developed at Seoul National University College of Medicine, is a computerized cognitive test that measures a child or adolescent's sustained and selective 
attention and impulsivity. ${ }^{13)}$ Although the ATA consists of a visual attention test and an auditory attention test, only a visual attention test was undertaken in this study, as described above. Regarding the visual attention ATA, the target or non-target stimulus generates 100 ms every 2 seconds for 15 minutes ( 5 minutes for five-year-olds, 10 minutes for six-year-olds). The target stimulus is a triangle; non-target stimuli are a circle and a square. Children press the button when target stimuli are presented. ATA is divided into three sections of 5 minutes. The rate of target stimuli changes in each section. The rate of the target is $22 \%$ in the first section, $50 \%$ in the second section, and $78 \%$ in the third section.

After completion, the ATA shows age-adjusted T-scores (mean=50, SD=10) of four indices, omission errors, commission errors, response time (RT), and SD of RTs overall (through the whole task), and for each section. An omission error occurs when a child fails to press the button when a target stimulus is presented. It reflects the child's sustained attention. ${ }^{15)} \mathrm{A}$ high T-score of omission error means that the number of omission errors was large, signaling poor attentivity. A commission error occurs when a child presses the button when non-target stimuli are present, indicating the child's impulsivity, poor self-regulation, and inhibitory control. ${ }^{15,16)}$ A high T-score of commission error means that commission errors occurred frequently, signaling poor control. The RT is the length of time from the onset of the target stimulus to pressing the button. The RT is said to reflect attention distraction and the response preparation of executive function. ${ }^{15,16)} \mathrm{High}$ T-scores of RTs mean that the RT is longer than the average RT. Thereby children are slow to react. The SD of RTs stands for the variation of the RT. Because this is attributed to the presence of numerous abnormally slow RT, The SD of RTs is typically interpreted as reflecting occasional lapses in attention. ${ }^{17,18)}$ The high T-score of SD of RTs means that the SD of RTs was large. In other words, the RT was not stable.

In actuality, ATA is also called "ADHD Diagnostic System (ADS)". ${ }^{13-15)}$ As its name suggests, ATA judges "ADHD", "suspicion of ADHD", and "normal" according to the performance of each participant. When one or more of four total T-scores (omission errors, commission errors, RT, and SD of RTs) exceeds 65 (over 1.5 SD), ATA judged the performance of the participant as "ADHD" range. When one or more of these total T-scores exceeds 60 (over 1.0 SD), ATA judged the participant as "the suspicion of ADHD" range.

\section{ADHD Rating Scale (ADHD-RS)}

ADHD-RS consists of 18 items that met DSM diagnostic criteria of ADHD, of which 9 items are related to inattention, 6 items are related to hyperactivity, and others are impulsivity. ${ }^{19)}$ There are a parent-complete version and a teacher-complete version, 18 items in both versions have a 4-point-Likert scale $(0=$ none, $1=$ mild, $2=$ moderate, $3=$ severe). As results of ADHD-RS, three scores (inattentive score, hyperactivity/impulsive score, total score) were calculated. A higher score indicates higher severity.

\section{Procedure}

First ATA was explained to a child and caregiver(s); then the child completed the visual attention test of ATA in a quiet room. Caregivers filled in ADHD-RS while the child worked on ATA.

Of the nine children treated with ATX, none had been prescribed medication for ADHD treatment: they were drug-naïve. Therefore, all children first completed the ATA in a medication-off condition, and then in a medication-on condition after at least 21 days (84.0 051.4 days; range, 21-205 days). Of 33 children treated with OROS-MPH, 10 children had not been on medication treatment (drugnaïve), 23 others had been treated with OROS-MPH. All 33 children of OROS-MPH group completed the ATA in a medication-off condition to match the paradigm for ATX and completed the medication-on condition after at least 14 days (43.3 \pm 49.9 days; range, 14-244 days). For the medication-off condition of 23 children who had taken OROS-MPH, we scheduled a longer than 24-hours period from the last time they had taken medication.

Caregivers filled in ADHD-RS on both clinical pictures of medication-off and medication-on. For drug-naïve children, we used ADHD-RS score caregivers filled in at their first hospital visit for the medication-off condition, and therefore before any treatment. For the medication-off condition of children who had been treated with OROSMPH, caregivers filled in the child's clinical pictures of losing the effects of OROS-MPH, for example after 12 hours from taking medicine or during a drug holiday.

\section{Statistical Analysis}

First, analysis of the ATA judgments was clarified. Furthermore, to determine if the rate of ATA judgments changed after medication, we compared the number of participants divided into three judgments ("ADHD", "suspicion of ADHD", and "normal") on each condition (medication on and off) with every subgroup, using exact tests. Secondly, discriminant analyses using a predictive 
model based on linear combinations of the predictor variables that provide the best discrimination between the groups. ${ }^{20)}$ Analyses were performed to ascertain whether the ADHD group could be differentiated based on T-scores of each index in each section (12 indexes; omission errors, commission errors, RT, and SD of RTs in three sections) for the medication-off condition. To conduct discriminant analysis, data of Korean control children were included for use in standardization.

Next, a 2 (subtype: inattentive, combined) $\times 2$ (medication: off, on) $\times 3$ (ATA section: Sections 1, 2, 3) mixed model analyses of variance (ANOVA) was undertaken for each index of ATA. From the perspective of clarifying medication efficacy, it is appropriate to include the medication type condition. However, only two children were diagnosed as inattentive ADHD and treated using ATX (Table 2). Therefore, the medication type condition was excluded from this research. The age condition was also excluded because of the use of an age-adjusted standard score as a dependent variable for analysis. Although suggestion of a relation between ATA and IQ was reported ${ }^{15)}$ and although some research found a relation between IQ and CPT performance, $^{21,22)}$ partial relation ${ }^{23)}$ and slight correlation ${ }^{24)}$ were also reported. Consequently, it is too early to be sure of a relation between IQ and CPT performance. Additionally, some children completed the WISC-III, whereas others completed the WISC-IV in this study. Therefore, the condition of IQ was also excluded from these analyses. A Greenhouse-Geisser (GG) correction for sphericity was applied to meet the requirements of compound symmetry.

Finally, for revealing correlations between the improvement of clinical symptoms and the improvement of ATA indices, correlation analyses were done. We defined the improvement of clinical symptom as the value obtained from subtracting the medication-on ADHD-RS score from medication-off ADHD-RS score, and defined the improvement of ATA as the value subtracting each T-score of medication-on from medication-off. We used IBM SPSS Statistics software ver. 22.0 (IBM Co., Armonk, NY, USA) for the statistical analyses described above.

\section{RESULTS}

\section{ATA Judgments and Discriminant Analyses}

The ATA judgments for ADHD diagnosis are presented in Table 3. ATA judged 12 out of $17(70.6 \%)$ children of the inattentive group and 20 out of $25(80.0 \%)$ children of the combined group as ADHD or ADHD suspicion in a medication-off condition. We administered an exact test by each group. Neither result was significant (inattentive $p=0.50$, combined $p=0.06$ ). Therefore, the rate of children who were judged as having ADHD or ADHD suspicion did not change after medication treatment. As a result of the discriminant analysis based on all T-scores, $89.6 \%$ of the control children and $69.0 \%$ of ADHD children were assigned correctly (Wilks' lambda $=0.79, \chi^{2}(12)=170.71, p$ $<0.001)$.

\section{ANOVA}

Regarding ANOVA results of commission errors, the main effects of subtype $\left(F[1,40]=10.33, p<0.01, \eta^{2}=0.11\right)$, medication $\left(F[1,40]=21.59, p<0.01, \eta^{2}=0.08\right)$, and section $\left(F[2,80]=9.83, p<0.01, \eta^{2}=0.03\right)$ were significant (Fig. 1). Inattentive groups showed lower T-scores than that of the combined group. The medication and section interaction was significant $\left(F[2,80]=4.46, p=0.02, \eta^{2}=0.01\right)$. Post hoc comparison with Bonferroni adjustments demonstrated that the T-scores of Sections 2 and 3 were higher than that of Section 1 in the medication-off condition (both $p<0.01$ ). The T-scores in all sections in the medication-on condition were lower than in the medication-off condition (all $p<0.01$ ).

Regarding Response Time, the main effect of the section was significant (GG: $F[1.55,61.90]=5.13, p=0.01$, $\eta^{2}=0.01$ ). Post hoc analysis with Bonferroni adjustments showed a T-score of Section 3 as lower than that of Section $2(p<0.05)$.

For SD of RTs, the main effect of subtype ( $F[1$, $\left.40]=4.50, p=0.04, \eta^{2}=0.04\right)$, medication $(F[1,40]=4.71$, $\left.p=0.04, \eta^{2}=0.02\right)$, and section $(F[2,80]=4.40, p=0.01$, $\left.\eta^{2}=0.02\right)$ were significant. T-scores of the Inattentive group and the medication-on condition were lower. In the section condition, T-scores of Section 3 were higher than those of Section 1 (Bonferroni adjusted post hoc, $p=0.04$ ).

Furthermore, no main effect or interaction with omission error was found. The mean of the T-score on each level was under 60.

Table 3. Results of Advanced Test of Attention (ATA) judgment

\begin{tabular}{ccccc}
\hline \multirow{2}{*}{$\begin{array}{c}\text { ADHD } \\
\text { subgroup }\end{array}$} & Medication & \multicolumn{3}{c}{ ATA Judgement } \\
\cline { 3 - 5 } & ADHD & $\begin{array}{c}\text { ADHD } \\
\text { suspicion }\end{array}$ & Non-ADHD \\
\hline Inattentive & Off & 6 & 6 & 5 \\
Combined & On & 4 & 4 & 9 \\
& Off & 17 & 3 & 5 \\
& On & 10 & 10 & 5 \\
\hline
\end{tabular}

ADHD, attention deficit/hyperactivity disorder. 

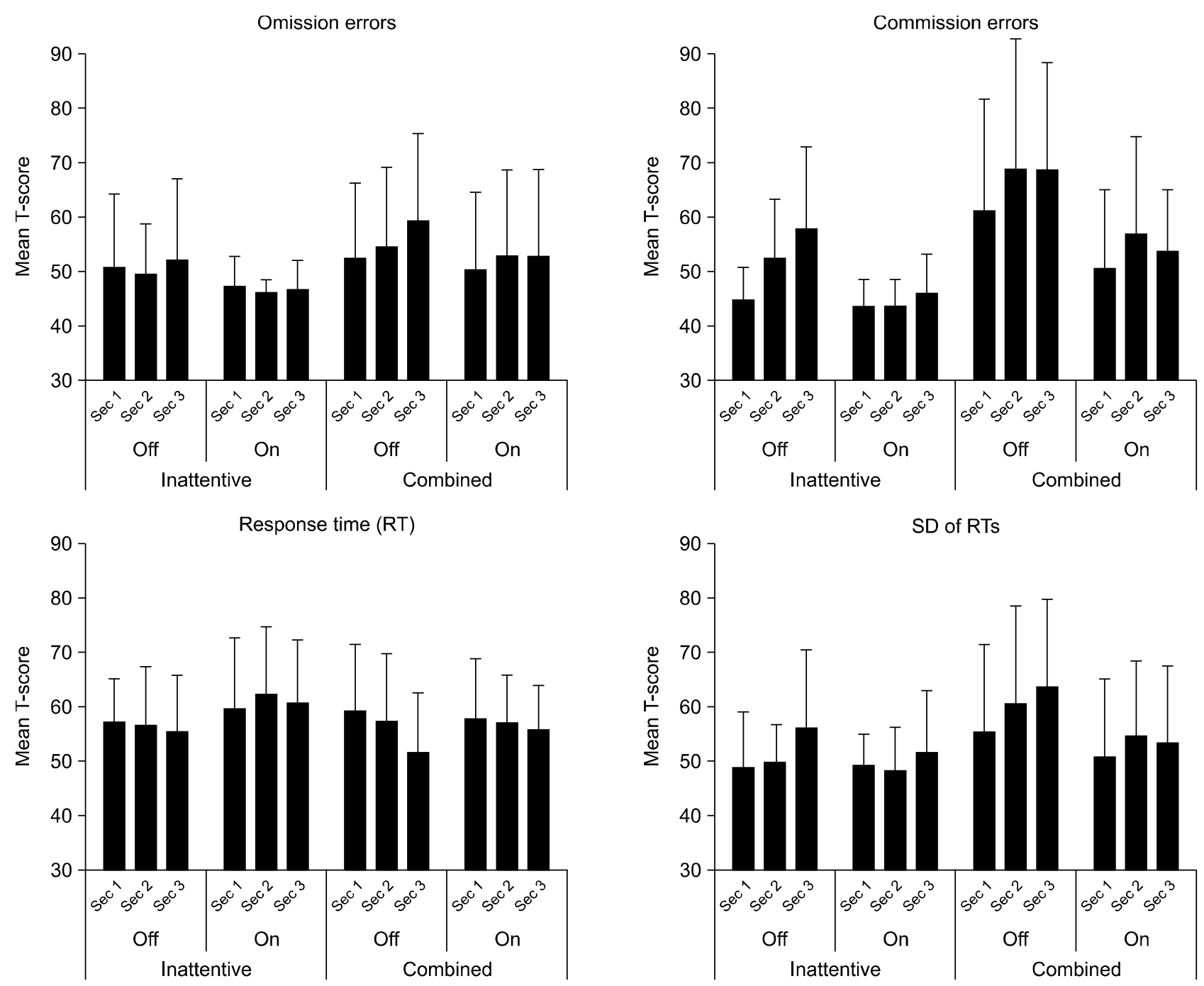

Fig. 1. Mean T-score of each index and each group.

Inattentive, inattentive ADHD group; combined, combined ADHD group; off, medication-off condition; on, medication-on condition; Sec 1, Section 1 of ATA; Sec 2, Section 2 of ATA; Sec 3, Section 3 of ATA.

ADHD, attention deficit/hyperactivity disorder; ATA, Advanced Test of Attention; SD, stadard deviation.

\section{Correlation Analysis}

For the score of ADHD-RS, 33 caregivers (14 from inattentive group, 19 from combined group) filled in all items of ADHD-RS; 9 were excluded because of missing values or difficulty in filling in the form in a medication-off condition (Fig. 2). To examine the effects of subtype (inattentive and combined), medication (off and on), and ADHD-RS subscale (inattention, hyperactivity/ impulsivity), a mixed ANOVA was done using scores of ADHD-RS as the dependent variable. Interaction among subtypes and medications and subscales was significant $\left(F[1,31]=5.72, p=0.02, \eta^{2}=0.01\right)$. Post hoc comparisons revealed that medication-off scores were higher than medication-on scores at all levels (all $p<0.01$ ).
A significant improvement in ADHD-RS scores was found during the follow-up period with OROS-MPH or ATX medication. Significant correlation was found between the improvement of inattentive symptom score and the improvement of omission errors in Section $3(r=0.40$, $p=0.02$ ), and between the improvement of hyperactivity/ impulsivity symptom score and the improvement of omission errors in Section $3(r=0.47, p<0.01)$ and of the SD of RTs in Section $1(r=0.43, p=0.01)$ as shown Table 4.

\section{Effects of Medication Treatment by MPH and ATX}

For confirmation of the treatment effect depending on the type of medicine, a 2 (type of medicine: MPH, ATX) $\times 2$ (medication: off, on) $\times 3$ (ATA section: sections $1,2,3$ ) 


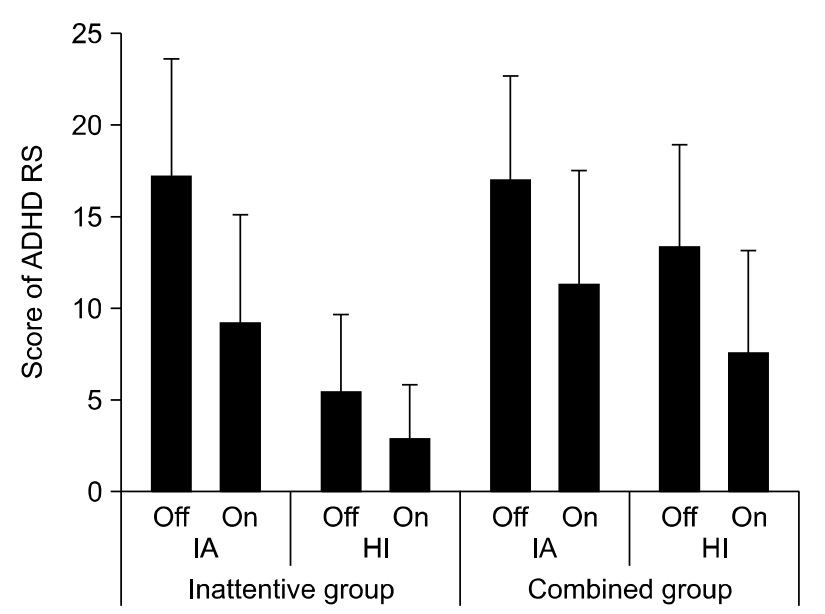

Fig. 2. Mean scores of Attention Deficit/Hyperactivity Disorder Rating Scale (ADHD-RS).

Off, medication-off condition; on, medication-on condition; IA, ADHD-RS inattention score; HI, ADHD-RS hyperactivity/impulsivity score.

mixed model ANOVAs for four indices was used. Results show that the main effects or interactions of type of medicine were not significant (all $p>0.05$ ).

\section{DISCUSSION}

\section{Validation of ATA Visual Attention Version in Japan}

For ADHD judgment of the ATA visual test, 12 out of $17(70.6 \%)$ children of the inattentive group and 20 out of $25(80.0 \%)$ children of the combined group were judged as having ADHD or were suspected of having ADHD in a medication-off condition, $69.0 \%$ of ADHD was correctly assigned for discriminant analysis. Corbett and Constantine $^{25)}$ reported that discriminate function analysis using omission and commission errors differentiated $66.7 \%$ of ADHD children from ASD and control with different CPT. Perugini et al ${ }^{26)}$ reported sensitivity of the CPT index as 0.67 . The discrimination values of this study replicate those of such previous studies, and reflect the validity of ATA.

For commission errors, the main effect of subtype and the interaction between medication and section was significant on ANOVA. Commission error, as described above, putatively reflects impulsivity, self-regulation, and inhibitory control. ${ }^{15,16)}$ Therefore, the higher T-score of the combined group than the inattentive group and mean T-score over 60 in the combined group reflected that ATA has sensitivity to impulsivity. Furthermore, the lower T-score of Section 1 in the medication-off condition means that the age-adjusted standard score increased as the trial proceeded; it might reflect the difficulty of sustained attention and the lessened ability to maintain a tonic state of
Table 4. Correlation between the improvement of clinical symptoms ${ }^{*}$ and the improvement of Advanced Test of Attention ${ }^{\dagger} \quad(n=33)$

\begin{tabular}{ccc}
\hline Variable & IA & $H$ \\
\hline Omission errors & & \\
Sec 1 & 0.07 & 0.05 \\
Sec 2 & 0.13 & -0.06 \\
Sec 3 & $0.40^{\ddagger}$ & $0.47^{\ddagger}$ \\
Commission errors & & \\
Sec 1 & -0.02 & 0.20 \\
Sec 2 & -0.10 & 0.13 \\
Sec 3 & 0.06 & 0.18 \\
RT Sec 1 & & \\
Sec 2 & 0.01 & 0.13 \\
Sec 3 & 0.09 & 0.07 \\
SD of RTs & 0.03 & -0.25 \\
Sec 1 & & \\
Sec 2 & 0.15 & $0.43^{\ddagger}$ \\
Sec 3 & 0.05 & 0.30 \\
\hline
\end{tabular}

*ADHD-RS score on medication-off minus one on medication-on; ${ }^{\dagger}$ T-score on medication-off minus one on medication.

IA, improvement score of Attention Deficit/Hyperactivity Disorder Rating Scale (ADHD-RS) inattention; HI, improvement score of ADHD-RS hyperactivity/impulsivity; Sec 1, Section 1 of ATA; Sec 2, Section 2 of ATA; Sec 3, Section 3 of ATA; RT, response time; SD, standard deviation.

$p<0.05$.

alertness during prolonged and sustained mental activity for ADHD. ${ }^{27)}$ Therefore, for commission error, these results were appropriate to assess ADHD.

In SD of RTs, the main effects of subtype, medication, and section were significant. As described above, the SD of RTs is said to reflect occasional lapses in attention. ${ }^{18)}$ Although many studies have treated RT variability, none of the differences between subtypes has been reported. ${ }^{28,29)}$ Some results indicated that it was related to the inattention and hyperactivity. ${ }^{30,31)}$ Therefore, it is said that this result replicates only a fraction of those earlier research results. For the main effect of section, the T-score of Section 3 was higher than that of Section 1 in this research, which also might reflect the difficulty of sustained attention of ADHD. ${ }^{27)}$

Furthermore, no main effect or interaction was found with omission error. The mean T-scores in all conditions were less than 60 . The rate of target increases as the task progresses, and opportunities that children have to press also increase. Therefore, omission error, failing to press, tended not to occur even in the mid-to-late section, which might reflect the difficulty of sustained attention.

As explained above, the result of ATA reflected features of ADHD and replicated previous studies, especially in commission errors and SD of RTs. Previous reports described that comorbid ASD and LD did not affect the performance of CPT. ${ }^{32-34)}$ Moreover, the number of each comorbid disorder was a small complex for adding to the factor of ANOVA. Therefore, we ignored the factor for 
each comorbid disorder. Consequently, the results of this study indicate validity and availability of ATA for assessment in Japan.

\section{Effects of Medication}

According to the ANOVA results of commission errors, medication decreased commission errors in the mid-to-late section. This result, showing that medication decreases commission errors, matches those of earlier studies. . $^{4,35,36)}$ No main effect or interaction related to medication effect on omission errors is shown in previous research using ATA (ADS), which is often said with MPH. ${ }^{14,36,37)}$ However, the pattern by which MPH decreases only commission errors, as in this study, was reported also from previous studies with MPH. ${ }^{38,39)}$ Furthermore, T-scores of omission error in this research were in the normal range in the medicationoff condition, so medication treatment effects in omission error were slight if they existed at all. Additionally, ANOVA shows medication effects on SD of RTs. This result demonstrates that medication made the RT stable. Although the mechanism of SD of RTs remains controversial and remains to be resolved in detail, ${ }^{18)}$ ANOVA results in this research replicated the medication effects on SD of RTs. ${ }^{17,35)}$ Those results proved that ATA can evaluate the efficacy of medication treatment.

Correlation analysis found a few significant correlations. Regarding improvement of omission error, significant correlation was found with improvements of clinical symptoms in Section 3. According to improvement of SD of RTs, significant correlation exists with improvement of hyperactivity/impulsivity in Section 1. Presumably, the difficulty of sustained attention as trials advanced was especially great in Section 3, so children whose ADHD traits are high might have failed to react to targets in Section 3 on medication-off condition. Thereby, the sensitivity to detect the improvement of ADHD traits measured by ADHD-RS might be high in Section 3. Furthermore, the relation between improvement of reaction time variation and hyperactivity/impulsivity was outstanding in the beginning section. This may mean that children whose hyperactivity/impulsivity was high showed difficulty reacting stably in the beginning of task under medication-off condition. And, the sensitivity to detect improvement of hyperactivity/impulsivity measured by ADHD-RS might be high in Section 1 with SD of RTs. For these reasons, the results of significant correlations were appropriate. Moreover, they reflect ADHD clinical pictures.

However, at this point, one important matter to mention is that ATA might assess the aspects of inattention and hy- peractivity/impulsivity that are difficult to elucidate by ADHD-RS. Although both in ATA and ADHD-RS, medication effects were observed clearly in this research, few significant correlations were found between the improvement of ATA indices and ADHD-RS. ATA assesses children's ADHD symptoms with a computer for 15 minutes, but not in a daily situation. The scores we obtain are objective behavioral indicators using a fixed paradigm and standard score. In ADHD-RS, however, caregivers filled it in based on their observation in daily living. The score includes a subjective viewpoint and might be incongruent if two individuals evaluate the same child's behavior or improvement. Therefore, correlation was not significant between the improvement of commission error, which reflected great medical improvement on ANOVA, and ADHD-RS. In sum, we would like to emphasize that child's symptoms or treatment effects are assessed from various perspectives, and ATA which can assess the symptoms of individual objectively with fixed paradigm and standard score is good way for it. Additionally, we think that examining whether ATA can measure "acute effect of medication (pre- and post-medication effect in a day)" makes it useful clinical tool, and this is an issue in the future.

\section{Effects of Medication Treatment between MPH and ATX}

Between MPH and ATX, no interaction or main effect was found. Some differential effects were found between MPH and ATX on executive function tasks that might depend on action mechanisms in previous studies, ${ }^{40,41)}$ which we will need to redefine, but the effect of medical treatment was shown clearly in ATA. Even considering the limitation that MPH and ATX were mixed for medication treatment and children treated with ATX were only nine in all, medical treatment for children with ADHD was shown to be worthwhile with Japanese children using ATA.

The aims of this study were to validate the visual attention version of ATA in Japan, and to evaluate the efficacy of medication (OROS-MPH and ATX) treatment using ATA. The feature of ADHD was reflected especially in the mid-to-late section with ANOVA. These results replicated those of previous studies. Although ATA was developed and standardized with Korean children and adolescents, results of this research proved the validity of ATA of the visual attention version in Japanese children with ADHD. Indices of ATA improved during medication treatment. A few significant correlations were found between the improvement of ATA and ADHD symptoms with medication treatment. Results suggest that ATA can evaluate 
the efficacy of medication treatment and suggest that indices of ATA reflect aspects of ADHD symptoms that are difficult to elucidate for ADHD-RS. Additionally, we were able to expand interpretation using standardized tasks. For example, significant medication effects are not apparent in omission errors because these indices were in the normal range for the medication-off condition. Such an interpretation with mean, $\mathrm{SD}$, and normal range is a great benefit of standardized tasks. These interpretations with ATA are useful for Japanese children with ADHD.

It is noteworthy that we must carefully interpret the ATA performance. Although ATA judges ADHD, the suspicion of ADHD or lack thereof, these ATA judgments should be interpreted as only one aspect of ADHD features. Moreover, we must regard the results of this research as reflecting the ADHD group trend. Therefore, some individuals might make many omission errors and few commission errors; some might show improved performance in the early section. Assessment should be done of various aspects and clinical features of each.

This research entails some limitations to be solved for better assessment for ADHD. First, we should analyze differences between MPH group and the ATX group. The different mechanisms of action between MPH and ATX might produce different performance with ATA paradigms. Second, because only a few girls were enrolled in this study, we did not include a gender factor in ANOVA. Therefore, it will be necessary to conduct this study with more children to clarify the effect of these factors. Third, many children were diagnosed with comorbid psychiatry disorders, and we did not include IQ scores in the analyses. These are natural and useful results for actual clinical situations, but it will be necessary conduct a study of the same paradigm with children who are diagnosed as having only ADHD and controlling IQ to establish a pure effect on medication effects to ADHD symptoms on ATA. Furthermore, indices of ATA appear to reflect aspects of ADHD symptoms that are difficult to be reflected on ADHD-RS. Better assessment is necessary to clarify differences between indices of these tools. Finally, many aspects of the paradigm require modification. For example, medication conditions should be counterbalanced between participants, and the same number of days between medication off and on condition was needed for MPH and ATX treatment. After surmounting these obstacles, ATA will be a better assessment tool for individuals with ADHD or other disorders.

\section{Acknowledgments}

We thank Akina Usui for assistance in subject recruitment, and Mika Yamazaki, Chisato Ohta, Riako Teshigawara, and Hitomi Suzuki for their excellent technical assistance in evaluating children.

This work was supported by Grants-in-Aid for Scientific Research (B) and Challenging Exploratory Research from the Ministry of Education, Culture, Sports, Science and Technology (MEXT) of Japan (KAKENHI: nos. 24300149 and 25560386 to AT). This work also was partially supported by a Grant-in-Aid for Scientific Research from the Japan-U.S. Brain Research Cooperation Program (no. 210201 to AT), as well as Research Grants from the University of Fukui to AT.

The funding organizations had no role in the design or conduct of the study, such as the collection, management, analysis, or interpretation of the data, or the preparation, review, or approval of the manuscript.

\section{REFERENCES}

1. American Psychiatric Association. Diagnostic and Statistical Manual of Mental Disorders: DSM-5. 5th ed. Washington, DC:American Psychiatric Association;2013.

2. Rubia K. "Cool" inferior frontostriatal dysfunction in attention-deficit/hyperactivity disorder versus "hot" ventromedial orbitofrontal-limbic dysfunction in conduct disorder: a review. Biol Psychiatry 2011;69:e69-e87.

3. Arnsten AF, Li BM. Neurobiology of executive functions: catecholamine influences on prefrontal cortical functions. Biol Psychiatry 2005;57:1377-1384.

4. Koechlin E, Summerfield C. An information theoretical approach to prefrontal executive function. Trends $\operatorname{Cog} n \mathrm{Sci}$ 2007;11:229-235.

5. Arnsten AF. Stimulants: Therapeutic actions in ADHD. Neuropsychopharmacology 2006;31:2376-2383.

6. Bidwell LC, McClernon FJ, Kollins SH. Cognitive enhancers for the treatment of ADHD. Pharmacol Biochem Behav 2011;99:262-274.

7. Rubia K, Alegria AA, Cubillo AI, Smith AB, Brammer MJ, Radua J. Effects of stimulants on brain function in attentiondeficit/hyperactivity disorder: a systematic review and meta-analysis. Biol Psychiatry 2014;76:616-628.

8. Rubia K, Halari R, Cubillo A, Mohammad AM, Brammer M, Taylor E. Methylphenidate normalises activation and functional connectivity deficits in attention and motivation networks in medication-naïve children with ADHD during a rewarded continuous performance task. Neuropharmacology 2009;57:640-652.

9. Schweren LJ, de Zeeuw P, Durston S. MR imaging of the effects of methylphenidate on brain structure and function in attention-deficit/hyperactivity disorder. Eur Neuropsychopharmacol 2013;23:1151-1164.

10. Wehmeier PM, Dittmann RW, Schacht A, Helsberg K, Lehmkuhl G. Morning and evening behavior in children and adolescents treated with atomoxetine once daily for attentiondeficit/hyperactivity disorder (ADHD): findings from two 24-week, open-label studies. Child Adolesc Psychiatry Ment 
Health 2009;3:5.

11. Riccio CA, Reynolds CR, Lowe P, Moore JJ. The continuous performance test: a window on the neural substrates for attention? Arch Clin Neuropsychol 2002;17:235-272.

12. Klee SH, Garfinkel BD. The computerized continuous performance task: a new measure of inattention. J Abnorm Child Psychol 1983;11:487-495.

13. Shin MS, Choi H, Kim H, Hwang JW, Kim BN, Cho SC. A study of neuropsychological deficit in children with obsessivecompulsive disorder. Eur Psychiatry 2008;23:512-520.

14. Lee SH, Song DH, Kim BN, Joung YS, Ha EH, Cheon KA, et al. Variability of response time as a predictor of methylphenidate treatment response in Korean children with attention deficit hyperactivity disorder. Yonsei Med J 2009;50: 650-655.

15. Park MH, Kweon YS, Lee SJ, Park EJ, Lee C, Lee CU. Differences in performance of ADHD children on a Visual and Auditory Continuous Performance Test according to IQ. Psychiatry Investig 2011;8:227-233.

16. Wang LJ, Huang YS, Chiang YL, Hsiao CC, Shang ZY, Chen CK. Clinical symptoms and performance on the Continuous Performance Test in children with attention deficit hyperactivity disorder between subtypes: a natural follow-up study for 6 months. BMC Psychiatry 2011;11:65.

17. Huang-Pollock CL, Karalunas SL, Tam H, Moore AN. Evaluating vigilance deficits in ADHD: a meta-analysis of CPT performance. J Abnorm Psychol 2012;121:360-371.

18. Tamm L, Narad ME, Antonini TN, O'Brien KM, Hawk LW Jr, Epstein JN. Reaction time variability in ADHD: a review. Neurotherapeutics 2012;9:500-508.

19. DuPaul GJ, Power TJ, Anastopoulos AD, Reid R. ADHD Rating Scale - IV: Checklists, norms, and clinical interpretation. New York:Guilford Press; 1998.

20. SPSS. IBM SPSS statistics base 22. Chicago, IL:SPSS Inc; 2013.

21. Jung RE, Yeo RA, Chiulli SJ, Sibbitt WL Jr, Brooks WM. Myths of neuropsychology: intelligence, neurometabolism, and cognitive ability. Clin Neuropsychol 2000;14:535-545.

22. Leeson VC, Barnes TR, Harrison M, Matheson E, Harrison I, Mutsatsa SH, et al. The relationship between IQ, memory, executive function, and processing speed in recent-onset psychosis: 1-year stability and clinical outcome. Schizophr Bull 2010;36:400-409.

23. Friedman NP, Miyake A, Corley RP, Young SE, Defries JC, Hewitt JK. Not all executive functions are related to intelligence. Psychol Sci 2006;17:172-179.

24. Naglieri JA, Goldstein S, Delauder BY, Schwebach A. Relationships between the WISC-III and the Cognitive Assessment System with Conners' rating scales and continuous performance tests. Arch Clin Neuropsychol 2005;20:385-401.

25. Corbett BA, Constantine LJ. Autism and attention deficit hyperactivity disorder: assessing attention and response control with the integrated visual and auditory continuous performance test. Child Neuropsychol 2006;12:335-348.

26. Perugini EM, Harvey EA, Lovejoy DW, Sandstrom K, Webb AH. The predictive power of combined neuropsychological measures for attention-deficit/hyperactivity disorder in children. Child Neuropsychol 2000;6:101-114.

27. Rapport MD, Orban SA, Kofler MJ, Friedman LM. Do programs designed to train working memory, other executive functions, and attention benefit children with ADHD? A meta-analytic review of cognitive, academic, and behavioral outcomes. Clin Psychol Rev 2013;33:1237-1252.

28. Geurts HM, Verté S, Oosterlaan J, Roeyers H, Sergeant JA. ADHD subtypes: do they differ in their executive functioning profile? Arch Clin Neuropsychol 2005;20:457-477.

29. Solanto MV, Gilbert SN, Raj A, Zhu J, Pope-Boyd S, Stepak $\mathrm{B}$, et al. Neurocognitive functioning in $A D / H D$, predominantly inattentive and combined subtypes. J Abnorm Child Psychol 2007;35:729-744.

30. Gómez-Guerrero L, Martín CD, Mairena MA, Di Martino A, Wang J, Mendelsohn AL, et al. Response-time variability is related to parent ratings of inattention, hyperactivity, and executive function. J Atten Disord 2011;15:572-582.

31. Buzy WM, Medoff DR, Schweitzer JB. Intra-individual variability among children with ADHD on a working memory task: an ex-Gaussian approach. Child Neuropsychol 2009; 15:441-459.

32. Nydén A, Niklasson L, Stahlberg O, Anckarsater H, Wentz $\mathrm{E}$, Rastam M, et al. Adults with autism spectrum disorders and ADHD neuropsychological aspects. Res Dev Disabil 2010;31:1659-1668.

33. Lundervold AJ, Stickert M, Hysing M, Sørensen L, Gillberg C, Posserud MB. Attention deficits in children with combined autism and ADHD: a CPT study. J Atten Disord 2012. doi: 10.1177/1087054712453168. [Epub ahead of print]

34. McGee RA, Clark SE, Symons DK. Does the Conners' Continuous Performance Test aid in ADHD diagnosis? J Abnorm Child Psychol 2000;28:415-424.

35. Epstein JN, Brinkman WB, Froehlich T, Langberg JM, Narad $\mathrm{ME}$, Antonini TN, et al. Effects of stimulant medication, incentives, and event rate on reaction time variability in children with ADHD. Neuropsychopharmacology 2011;36: 1060-1072.

36. Lee SI, Hong SD, Kim SY, Kim EJ, Kim JH, Kim JH, et al. Efficacy and tolerability of OROS methylphenidate in Korean children with attention-deficit/hyperactivity disorder. Prog Neuropsychopharmacol Biol Psychiatry 2007;31:210216.

37. Solanto MV. Neuropsychopharmacological mechanisms of stimulant drug action in attention-deficit hyperactivity disorder: a review and integration. Behav Brain Res 1998; 94:127-152.

38. Johnson KA, Barry E, Bellgrove MA, Cox M, Kelly SP, Dáibhis A, et al. Dissociation in response to methylphenidate on response variability in a group of medication naïve children with ADHD. Neuropsychologia 2008;46:1532-1541.

39. Barkley RA, Murphy KR, O'Connell T, Connor DF. Effects of two doses of methylphenidate on simulator driving performance in adults with attention deficit hyperactivity disorder. J Safety Res 2005;36:121-131.

40. Kratz O, Studer P, Baack J, Malcherek S, Erbe K, Moll GH, et al. Differential effects of methylphenidate and atomoxetine on attentional processes in children with ADHD: an event-related potential study using the Attention Network Test. Prog Neuropsychopharmacol Biol Psychiatry 2012; 37:81-89.

41. Yildiz O, Sismanlar SG, Memik NC, Karakaya I, Agaoglu B. Atomoxetine and methylphenidate treatment in children with ADHD: the efficacy, tolerability and effects on executive functions. Child Psychiatry Hum Dev 2011;42:257-269. 